\title{
A Discrete Least Squares Method
}

\author{
By Peter H. Sammon*
}

\begin{abstract}
We consider a discrete least squares approximation to the solution of a two-point boundary value problem for a $2 m$ th order elliptic operator. We describe the approximation space of piecewise polynomials and devise a Gaussian quadrature rule that is suitable for replacing the integrals in the usual least squares method. We then show that if the quadrature rule is of sufficient accuracy, the optimal order of convergence is obtained.
\end{abstract}

1. Introduction. Let $a<b$. We shall consider a scheme for finding an approximate solution to the following uniformly strongly elliptic boundary value problem:

$$
\begin{aligned}
& L u(x)=\sum_{r, s=0}^{m}(-1)^{r} D^{r}\left(a_{r s}(x) D^{s} u(x)\right)=f(x) \quad \text { on }(a, b), \\
& D^{s} u(a)=D^{s} u(b)=0 \quad \text { for } 0 \leqslant s \leqslant m-1,
\end{aligned}
$$

where we require that $a_{r s}(x)=a_{s r}(x)$ for $0 \leqslant r, s \leqslant m$. For simplicity, we shall assume that the coefficient functions belong to $C^{\infty}[a, b]$. We shall also assume that $L$ has a trivial null space so that unique solutions are guaranteed.

The approximate solution will be found by a discretized least squares method that utilizes a Gaussian quadrature rule for integral evaluation. The Gaussian rule will be studied because it provides a high degree of precision in general situations, at minimal computational expense. We will describe the method and show which Gaussian quadrature rules achieve the optimal order of accuracy when used with approximation spaces of piecewise polynomials.

We refer the reader to Ciarlet and Raviart [2] for a thorough description of the discretized Galerkin method in $\mathbf{R}^{d}$.

2. Preliminaries. We let $W^{r}(a, b), r$ a nonnegative integer, denote the usual $L^{2}$. based Sobolev spaces on $(a, b)$ and

$$
W_{o}^{m}(a, b)=\left\{g \in W^{m}(a, b): D^{s} g(a)=D^{s} g(b)=0 \text { for } 0 \leqslant s \leqslant m-1\right\} .
$$

We also let $V^{r}(a, b)=W^{r}(a, b) \cap W_{o}^{m}(a, b)$ and note that $W^{r}(a, b)\left(\right.$ or $\left.V^{r}(a, b)\right)$ can be normed by

$$
\|\cdot\|_{r,(a, b)}=\left(\sum_{s=0}^{r} \int_{a}^{b}\left|D^{s}(\cdot)(x)\right|^{2} d x\right)^{1 / 2} .
$$

We shall suppress the $(a, b)$ in the notation in what follows.

Received February 7, 1975.

AMS (MOS) subject classifications (1970). Primary 62 L10; Secondary 65D30.

* This paper is based on the author's Master's thesis written under the supervision of Dr. J. M. Varah at the University of British Columbia.

Copyright $\odot 1977$, American Mathematical Society 
We note that our assumptions on $L$ give us the following regularity result. If $r$ is a nonnegative integer, then there is a constant $C=C(r)>0$ so that

$$
(1 / C)\|g\|_{r+2 m} \leqslant\|L g\|_{r} \leqslant C\|g\|_{r+2 m} \text { for all } g \in V^{r+2 m} .
$$

We now introduce some notation which will aid us in our description of the approximation space $S^{h}$. If $\Delta$ is the partition on $[a, b]$ given by

$$
\Delta: a=x_{0}<x_{1}<\cdots<x_{N}<x_{N+1}=b
$$

and $n$ is a positive integer, we let $\mathbf{P}^{n}(\Delta)$ denote the set of functions on $[a, b]$ whose restriction to each open subinterval of $\Delta$ is a polynomial of degree $(n-1)$. If $r$ is a nonnegative integer, we let

$$
\|\cdot\|_{r, \Delta}=\left(\sum_{i=0}^{N} \sum_{s=0}^{r} \int_{x_{i}}^{x_{i+1}}\left|D^{s}(\cdot)(x)\right|^{2} d x\right)^{1 / 2} .
$$

We assume that $S^{h}$ satisfies the following conditions:

(1) $S^{h} \subset \mathbf{P}^{n}(\Delta) \cap C^{z}[a, b] \cap W_{o}^{m}$ where $n>2 m, z \geqslant 2 m-1$ and $\Delta=\left\{x_{i}\right\}_{i=0}^{N}$.

(2) We have that

$$
\inf _{\chi \in S}\|g-\chi\|_{2 m} \leqslant C h^{n-2 m}\|g\|_{n} \text { for all } g \in V^{n},
$$

where $h=\max \left\{\left(x_{i+1}-x_{i}\right): 0 \leqslant i \leqslant N\right\}$ and $C$ is independent of $g$ and $h$.

We note that $C$ is allowed to depend on an upper bound $\alpha$ for the mesh ratio, given by $\left(h / \min \left\{\left(x_{i+1}-x_{i}\right): 0 \leqslant i \leqslant N\right\}\right)$.

In the future, $C$ will denote a generic positive constant that depends on $L$ and all of the parameters that have been mentioned, except $h$.

Later we will need the following fact. If $0 \leqslant r \leqslant s \leqslant n-1$ and $\chi \in \mathbf{P}^{n}(\Delta)$, then

$$
\|\chi\|_{s, \Delta} \leqslant C h^{r-s}\|\chi\|_{r, \Delta}
$$

Thus, we have inverse properties in $S^{h}$. We also have

Proposition 2.1. If $n-2 m \geqslant 2 m, 0 \leqslant r \leqslant n-2 m$ and $v \in W^{r}$, then

$$
\inf _{\chi \in S^{h}}\|v-L \chi\|_{0} \leqslant C h^{r}\|v\|_{r}
$$

Proof. Estimates of (2.3) can be obtained for $r=0$ and $r=n-2 m$ using (2.1) and (2.2). The result then follows by interpolation.

3. The Least Squares Method. For the sake of comparison, we include the following easily proven estimates. If $A: V^{2 m} \rightarrow S^{h}$ is defined as the projection orto $S^{h}$ in the $\|L(\cdot)\|_{0}$ norm (see (2.1)), then $A g$, where $g \in V^{2 m}$, is characterized by

$$
b(A g, \chi) \equiv \int_{a}^{b} L(A g) \cdot L \chi d x=\int_{a}^{b} L g \cdot L \chi d x=b(g, \chi) \quad \text { for all } \chi \in S^{h}
$$

and, with $r=\min (n, 2 n-4 m)$, satisfies the following estimates:

$$
\|g-A g\|_{2 m} \leqslant C h^{n-2 m}\|g\|_{n}, \quad\|g-A g\|_{0} \leqslant C h^{r}\|g\|_{n} .
$$


4. Gaussian Quadrature. Let $K>0$ and fix some interval $\left(x_{i}, x_{i+1}\right)$ in $\Delta$. Let $\left\{z_{i j}\right\}_{j=1}^{K}$ be the roots of the $K$ th Legendre polynomial on $\left(x_{i}, x_{i+1}\right)$. These are the Gaussian quadrature nodes in $\left(x_{i}, x_{i+1}\right)$. It is well known that there exist (unique) weights $w_{i j}>0,1 \leqslant j \leqslant K$, which give a quadrature sum on $\left(x_{i}, x_{i+1}\right)$ that is precise for polynomials of degree $(2 K-1)$.

Using these nodes and weights on each subinterval, we can devise a composite quadrature sum on $[a, b]$ and thereby define approximations to the quantities $\|\cdot\|_{r, \Delta}$ and $b(\cdot, \cdot)$. For $r$ a nonnegative integer, let

$$
\begin{gathered}
\|\cdot\|_{r, \Delta}^{\prime}=\left(\sum_{i=0}^{N} \sum_{j=1}^{K} \sum_{s=0}^{r} w_{i j}\left|D^{s}(\cdot)\left(z_{i j}\right)\right|^{2}\right)^{1 / 2}, \\
b^{\prime}(\cdot, \cdot)=\left(\sum_{i=0}^{N} \sum_{j=1}^{K} w_{i j} L(\cdot)\left(z_{i j}\right) \cdot L(\cdot)\left(z_{i j}\right)\right) .
\end{gathered}
$$

THEOREM 4.1. (1) If $g_{1}$ and $g_{2}$ are suitably smooth, then

$$
\left|\left(b-b^{\prime}\right)\left(g_{1}, g_{2}\right)\right| \leqslant C h^{2 K}\left\|g_{1}\right\|_{2 K+2 m, \Delta}\left\|g_{2}\right\|_{2 K+2 m, \Delta} \cdot
$$

(2) If $\chi_{1}, \chi_{2} \in S^{h}$ and $r=2 K-2(n-2 m)+2$, then

$$
\left|\left(b-b^{\prime}\right)\left(\chi_{1}, \chi_{2}\right)\right| \leqslant C h^{r}\left\|\chi_{1}\right\|_{2 m}\left\|\chi_{2}\right\|_{2 m} .
$$

Proof. To demonstrate part (1), we expand $L g_{1} \cdot L g_{2}$ in a $2 K$-term Taylor series with integral remainder about the left endpoint of each subinterval in $\Delta$. Then we use the precision of the Gaussian rule for polynomials of degree $(2 K-1)$ and an estimate of the remainder to obtain (4.1). Part (2) follows from part (1) and inverse properties.

This result will be used later to choose $K$.

5. The Discretized Least Squares Method. We will now use our quadrature sum to define an operator related to the operator $A$ mentioned above.

Let $A^{\prime}: V^{n} \rightarrow S^{h}$ be defined for each $g \in V^{n}$ as the function in $S^{h}$ that minimizes $\|L(\cdot-g)\|_{0, \Delta}^{\prime}$ over $S^{h}$. The next result will show that $A^{\prime}$ is well defined by this procedure and that it is characterized by the equation $b^{\prime}\left(g-A^{\prime} g, \chi\right)=0$, for all $\chi \in S^{h}$.

THEOREM 5.1. If $K=n-2 m$ and $h$ is sufficiently small, then $A^{\prime}$ is well defined and if $g \in V^{n}$, we have that

$$
\left\|g-A^{\prime} g\right\|_{2 m} \leqslant C h^{n-2 m}\|g\|_{n} .
$$

Proof. Fix $\chi \in S^{h}$. Then if $h$ is sufficiently small, (2.1) and (4.2) show that

$$
b^{\prime}(\chi, \chi)=b(\chi, \chi)+\left(b^{\prime}-b\right)(\chi, \chi) \geqslant\|L \chi\|_{0}^{2}-C h^{2}\|\chi\|_{2 m}^{2} \geqslant C\|\chi\|_{2 m}^{2} .
$$

Similarly, $b^{\prime}(\chi, \chi) \leqslant C\|\chi\|_{2 m}^{2}$. Thus, $b^{\prime}(\cdot, \cdot)$ is an inner product on $S^{h}$. Hence, $A^{\prime}$ is well defined and is characterized by the equation noted above.

We now choose the $\chi \in S^{h}$ at which the infimum in (2.2) is attained.

We will first show that 


$$
\left\|\chi-A^{\prime} g\right\|_{2 m} \leqslant C\|\chi-g\|_{2 m, \Delta}^{\prime} \text {. }
$$

Since $b^{\prime}\left(g-A^{\prime} g, \chi\right)=0$, Schwarz's inequality (we recall that $w_{i j}>0$ ) and simple estimates yield the following:

$$
\begin{aligned}
C\left\|\chi-A^{\prime} g\right\|_{2 m}^{2} & \leqslant b^{\prime}\left(\chi-A^{\prime} g, \chi-A^{\prime} g\right)=b^{\prime}\left(\chi-A^{\prime} g, \chi-g\right) \\
& \leqslant\left[b^{\prime}\left(\chi-A^{\prime} g, \chi-A^{\prime} g\right)\right]^{1 / 2}\left[b^{\prime}(\chi-g, \chi-g)\right]^{1 / 2} \\
& \leqslant C\left\|\chi-A^{\prime} g\right\|_{2 m}\|\chi-g\|_{2 m, \Delta}^{\prime} .
\end{aligned}
$$

This gives (5.2).

For each $0 \leqslant i \leqslant N$, we let $E_{i} g$ be the unique polynomial of degree $(n-1)$ on $\left(x_{i}, x_{i+1}\right)$ that satisfies $D^{2 m} E_{i} g\left(z_{i j}\right)=D^{2 m} g\left(z_{i j}\right)$ for $1 \leqslant j \leqslant K=n-2 m$ and $D^{s} E_{i} g\left(z_{i 1}\right)=D^{s} g\left(z_{i 1}\right)$ for $0 \leqslant s \leqslant 2 m-1$. If $E g \in \mathbf{P}^{n}(\Delta)$ is the piecewise polynomial on $[a, b]$ made up of the $\left\{E_{i} g\right\}_{i=0}^{N}$, then as in Ciarlet and Raviart [1], one can show that

$$
\|g-E g\|_{r, \Delta} \leqslant C h^{n-r}\|g\|_{n} \text { for } 0 \leqslant r \leqslant n .
$$

By expanding $\left|D^{s}(g-E g)\right|^{2}(0 \leqslant s \leqslant 2 m-1)$ in a one-term Taylor series about the left endpoint of each subinterval and using (5.3), we find that

$$
\left|\left(\|g-E g\|_{2 m-1, \Delta}^{\prime}\right)^{2}-\left(\|g-E g\|_{2 m-1, \Delta}\right)^{2}\right| \leqslant C h^{2(n-2 m)+1}\|g\|_{n}^{2} .
$$

But by our choice of $E g,\|g-E g\|_{2 m, \Delta}^{\prime}=\|g-E g\|_{2 m-1, \Delta}^{\prime}$. Thus, by (5.3) and the last estimate, we have

$$
\begin{aligned}
\|g-E g\|_{2 m, \Delta}^{\prime} & \leqslant C\left(h^{2(n-2 m)+1}\|g\|_{n}^{2}+\|g-E g\|_{2 m-1, \Delta}^{2}\right)^{1 / 2} \\
& \leqslant C h^{n-2 m}\|g\|_{n} .
\end{aligned}
$$

We now use another Taylor series expansion, as in the proof of (4.1), to show that

$$
\begin{aligned}
& \left|\left(\|\chi-E g\|_{2 m, \Delta}^{\prime}\right)^{2}-\left(\|\chi-E g\|_{2 m, \Delta}\right)^{2}\right| \\
& \quad \leqslant C h^{2 K}\|\chi-E g\|_{2 K+2 m, \Delta}^{2}=C h^{2 K}\|\chi-E g\|_{n-1, \Delta}^{2} \leqslant C h^{2}\|\chi-E g\|_{2 m, \Delta}^{2} .
\end{aligned}
$$

Note that inverse properties and the low polynomial degrees proved useful.

We now can complete the proof. We use (5.2)-(5.5) to find that

$$
\begin{aligned}
\left\|g-A^{\prime} g\right\|_{2 m} & \leqslant\|g-\chi\|_{2 m}+C\|\chi-g\|_{2 m, \Delta}^{\prime} \\
& \leqslant C h^{n-2 m}\|g\|_{n}+C\|\chi-E g\|_{2 m, \Delta}^{\prime}+C\|E g-g\|_{2 m, \Delta}^{\prime} \\
& \leqslant C h^{n-2 m}\|g\|_{n}+C\|\chi-E g\|_{2 m, \Delta} \leqslant C h^{n-2 m}\|g\|_{n} .
\end{aligned}
$$

We will now prove an $L^{2}$ error estimate for $g-A^{\prime} g$. Note that the hypotheses given below demand more continuity than those of the last result.

THEOREM 5.2. If $g \in W^{2 n-2 m}, K=n-2 m$ and $h$ is sufficiently small, then

$$
\left\|g-A^{\prime} g\right\|_{0} \leqslant C h^{\min (n, 2 n-4 m)}\|g\|_{2 n-2 m} .
$$

Proof. Let $v \in V^{2 m}$ satisfy $L v=\left(g-A^{\prime} g\right) /\left\|g-A^{\prime} g\right\|_{0}$. Then for any $\chi \in S^{h}$, we have that 


$$
\begin{aligned}
\left\|g-A^{\prime} g\right\|_{0} & =\int_{a}^{b}\left(g-A^{\prime} g\right)(x) \cdot L v(x) d x \\
& =\int_{a}^{b}\left(L\left(g-A^{\prime} g\right)(x)\right)(v-L \chi)(x) d x+\left(b-b^{\prime}\right)\left(g-A^{\prime} g, \chi\right) \\
& \leqslant C\left\|g-A^{\prime} g\right\|_{2 m}\|v-L \chi\|_{0}+\left|\left(b-b^{\prime}\right)\left(g-A^{\prime} g, \chi\right)\right| .
\end{aligned}
$$

Also, if $w \in V^{4 m}$ solves $L w=v$, then $\|w\|_{4 m} \leqslant C$.

Using (2.2), (5.1) and inverse properties, one can obtain the following estimate:

$$
\left\|g-A^{\prime} g\right\|_{2 K+2 m, \Delta} \leqslant C\|g\|_{2 n-2 m} .
$$

We will need this result later.

Say $2 m>n-2 m$. Since $V^{4 m} \subset V^{n}$, we can use (2.2) to find a $\chi \in S^{h}$ that satisfies $\|w-\chi\|_{2 m} \leqslant C h^{n-2 m}$. Then (2.1) shows that

$$
\|v-L \chi\|_{0} \leqslant C h^{n-2 m} \text {. }
$$

Since $w \in V^{n}$, we can proceed as in the last proof and define a piecewise polynomial $E w \in \mathbf{P}^{n}(\Delta)$ which satisfies an estimate like (5.3). Then using inverse properties and this polynomial, one can easily show that

$$
\|\chi\|_{2 K+2 m, \Delta}=\|\chi\|_{n-1, \Delta} \leqslant C .
$$

Taking this $\chi$ in (5.7) and using (4.1), (5.1) and (5.8)--(5.10) we obtain the result for $2 m>n-2 m$.

Say $2 m \leqslant n-2 m$. We can use (2.3) to choose a $\chi \in S^{h}$ that satisfies $\|v-L \chi\|_{0}$ $\leqslant C h^{2 m}$. We then can construct a piecewise polynomial $E w$, of degree $(4 m-1)$ on each subinterval of $\Delta$, so that the $2 m$ th order derivatives of $w$ and $E w$ match at $2 m$ Gaussian points. We have, via the techniques of [1], that

$$
\|w-E w\|_{r, \Delta} \leqslant C h^{4 m-r} \text { for } 0 \leqslant r \leqslant 4 m .
$$

Then using (5.11) and inverse properties, we see that

$$
\|\chi\|_{4 m, \Delta} \leqslant\|\chi-E w\|_{4 m, \Delta}+\|E w-w\|_{4 m, \Delta}+\|w\|_{4 m, \Delta} \leqslant C .
$$

If we take this $\chi$ in (5.7), we can estimate the first term using (2.3) and (5.1). We can use (4.1) and inverse properties to estimate the second term as follows:

$$
\left|\left(b-b^{\prime}\right)\left(g-A^{\prime} g, \chi\right)\right| \leqslant C h^{2 n-4 m-n+1+4 m}\left\|g-A^{\prime} g\right\|_{2 K+2 m, \Delta}\|\chi\|_{4 m, \Delta} .
$$

Then (5.8) and (5.12) complete the proof.

Using the techniques of this section, it is possible to estimate $\left\|D^{s}\left(g-A^{\prime} g\right)\right\|_{0, \Delta}$ for $0 \leqslant s \leqslant n$.

We note that to use these methods on (1.1) in practice, one must find the solution of the following linear system:

$$
\sum_{r=1}^{d}\left(\sum_{i=0}^{N} \sum_{j=1}^{K} w_{i j} L \chi_{r}\left(z_{i j}\right) L \chi_{s}\left(z_{i j}\right)\right) C_{r}=\left(\sum_{i=0}^{N} \sum_{j=1}^{K} f\left(z_{i j}\right) L \chi_{s}\left(z_{i j}\right)\right) \quad \text { for } 1 \leqslant s \leqslant d,
$$


where $\left\{\chi_{r}\right\}_{r=1}^{d}$ is a basis for $S^{h}$ and $A^{\prime} u=\sum_{r=1}^{d} C_{r} \chi_{r}$ is the discrete least squares approximation to the solution of $L u=f$. If $f \in W^{2 n-4 m}, A^{\prime} u$ satisfies the estimates given by (5.1) and (5.6). Thus, upon examination of Section 3, we see that if $K=n$ $-2 m$ Gaussian nodes are used in each subinterval of $\Delta$, the discretized least squares method obtains the optimal order of accuracy. We refer the reader to Russell and Varah [3] for a discussion of the computational merits of the discretized least squares method.

Department of Mathematics

Cornell University

White Hall

Ithaca, New York 14853

1. P. G. CIARLET \& P.-A. RAVIART, "General Lagrange and Hermite interpolation in $\mathbf{R}^{n}$ with applications to finite element methods," Arch. Rational Mech. Anal., v. 46, 1972, pp. 177199. MR 49 \#1730.

2. P. G. CIARLET \& P.-A. RAVIART, "The combined effects of curved boundaries and numerical integration in isoparametric methods," The Mathematical Foundations of the Finite Element Method with Applications to Partial Differential Equations (A. K. Aziz, Editor), Academic Press, New York and London, 1972, pp. 409-474.

3. R. D. RUSSELL \& J. M. VARAH, "A comparison of global methods for linear two-point boundary value problems," Math. Comp., v. 29, 1975, pp. 1007-1019. 